\title{
A NEW APPROACH TO THE SPATIO-TEMPORAL PATTERN IDENTIFICATION IN NEURONAL MULTI-ELECTRODE REGISTRATIONS
}

\author{
Federico Esposti, Jacopo Lamanna and Maria Gabriella Signorini \\ Dipartimento di Bioingegneria, Politecnico di Milano, p.zza Leonardo da Vinci , 20133, Milano, Italy, \\ phone: + 39- 02-23999503, fax: + 39-02-23999508, e-mail: federico.esposti@polimi.it
}

\begin{abstract}
A lot of methods were created in last decade for the spatio-temporal analysis of multi-electrode array (MEA) neuronal data sets. All these methods were implemented starting from a channel to channel analysis, with a great computational effort and onerous spatial pattern recognition task.

Our idea is to approach the MEA data collection from a different point of view, i.e. considering all channels simultaneously. We transform the $2 D$ plus time MEA signal in a mono-dimensional plus time signal and elaborate it as a normal $1 D$ signal, using the SpaceAmplitude Transform method.

This geometrical transformation is completely invertible and allows to employ very fast processing algorithms.
\end{abstract}

Key-words: MEA technology, spike, Burst, spatiotemporal clustering, ACF, CCF.

\section{INTRODUCTION}

In last years a lot of efforts were spent in facing the problem of the analysis of the enormous amount of data coming from neuronal population electric recordings from Multi-electrode array (MEA) devices $[1,2]$. In fact, for a standard recording, made with 60 electrodes for 6 hours at tens of $\mathrm{kHz}$, we face with orders of $10^{10}$ samples. Starting from the assumption of the repetitiveness of the neuronal spike shape, the data set is simplified as a set of point process (one for each electrode) allowing the reduction of samples of about one magnitude order (or more, depending from the desired resolution) [3, 4].

This simplification, although very important, doesn't allow however a direct facing with the problem. We recall that, from the physical point of view, the problem is $(2+1)$ dimensional, i.e. is a $2 \mathrm{D}$ (recording MEA) plus time dimensional situation. A direct approach would be performed using image processing techniques, excessively heavy from the computation point of view facing this data amount. The actual standard approach is performed analyzing separately each channel (or signal component if a PCA analysis is previously done, in order to separate different neuron components) and looking for statistical or morphological similarities among channels [5-7].

The use of these techniques allowed to investigate a multitude of fundamental statistical properties of neuronal networks but made very difficult and onerous the inter-channel comparison.

A partial inter-channel analysis was proposed in last years by means of pattern recognition algorithms [813]. These techniques look for repetitive inter-channel spike sequences with complex pattern recognition algorithm, using, for example, neural networks [14] and/or Independent Component analysis [15]. They produced important results, in particular causing interesting discussion on the analysis jitter time, but at cost of a huge computational charge.

The purpose of our work was to produce an analysis method optimized for the inter-channel analysis, i.e. able to highlight the spatio-temporal relationships between MEA channels, with a very low computational cost.

\section{MATERIALS AND METHODS}

Our new approach to the data analysis is based on the concept of Space-Amplitude Transform. The method allows to approach an intrinsically 2D plus time data, i.e. time recordings from a $2 \mathrm{D}$ electrode array, as $1 \mathrm{D}$ plus time signals in order to speed up and make simpler the data analysis.

The Space-Amplitude Transform, $\mathbf{A}(\boldsymbol{s}, \boldsymbol{R})$, is a geometric transform that projects from a $2 \mathrm{D}$ domain set $s(x, y, t)$, e.g. the usual Raster plot, to a 1D image set $\boldsymbol{I}(\boldsymbol{r}, \boldsymbol{t})=\boldsymbol{A}(\mathbf{s}, \boldsymbol{R})$, exploiting an Arrangement algorithm $\boldsymbol{R}$. In the domain set $s(x, y, t)$, i.e. in the Raster plot, a specific spike is coded in terms of $0-1$ event (as said in the INTRODUCTION) that is located in a spacetemporal coordinate $(\mathrm{x}, \mathrm{y}, \mathrm{t})$, i.e. in a specific MEA channel (or trace if a PCA analysis is implemented) at a specific temporal instant (function of the system resolution).

The $\boldsymbol{A}(\boldsymbol{s}, \boldsymbol{R})$ transform arranges the MEA channels in a $1 \mathrm{D}$ list $\boldsymbol{r}$, according to a chosen arrangement algorithm $\boldsymbol{R}$ (with $\boldsymbol{r}=\mathrm{R}(\mathrm{x}, \mathrm{y})$ ), and associates each element of the list $\boldsymbol{r}$ to an ordinate numeric value (Fig.1). This operation allows building up a new signal $\mathbf{I}(\boldsymbol{r}, \boldsymbol{t})$ that 
associate to a domain space coordinate $(\bar{x}, \bar{y})$ a specific numeric value $(\bar{r})$, i.e. an image amplitude. So, in the image set $\boldsymbol{I}(\boldsymbol{r}, \boldsymbol{t})$, a spike $\boldsymbol{s}(\bar{x}, \bar{y}, \bar{t})$ of the raster plot is the point $(\bar{r}, \bar{t})$ of a new 1D function.

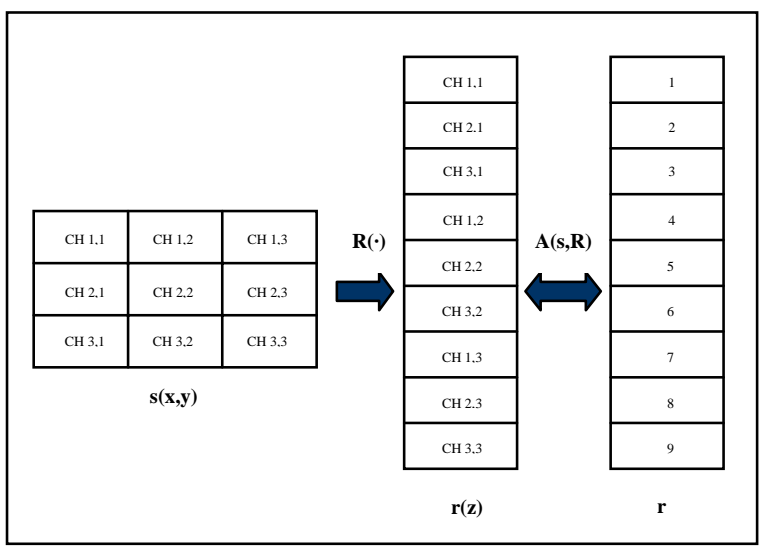

Fig.1: Graphical example of the role of the arrangement algorithm $R$ and the Space-Amplitude Transform A(s,R) with a $3 \times 3$ domain matrix. In this case the transformation leads to a traditional channel arrangement (see text).

In a graphic representation, the output of the SpaceAmplitude Transform can be imaged as the interpolating function of the Raster plot that locally assumes a value assigned on the basis of the Arrangement algorithm (Fig.2).

The shape of the resulting signal, as the Raster plot, depends from the arrangement algorithm and from the resolution used in the transformation but represents the spatial and temporal activity of the network in its entirety. The possibility to completely reconstruct the physic meaning of the further elaboration of this signal is granted by the biunivocality of the Space-Amplitude transform.

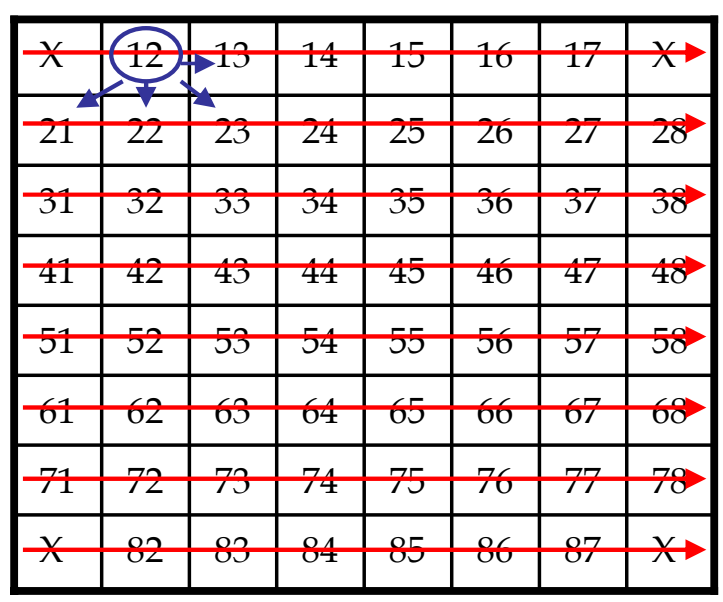

TRADITIONAL, ARRANGEMENT

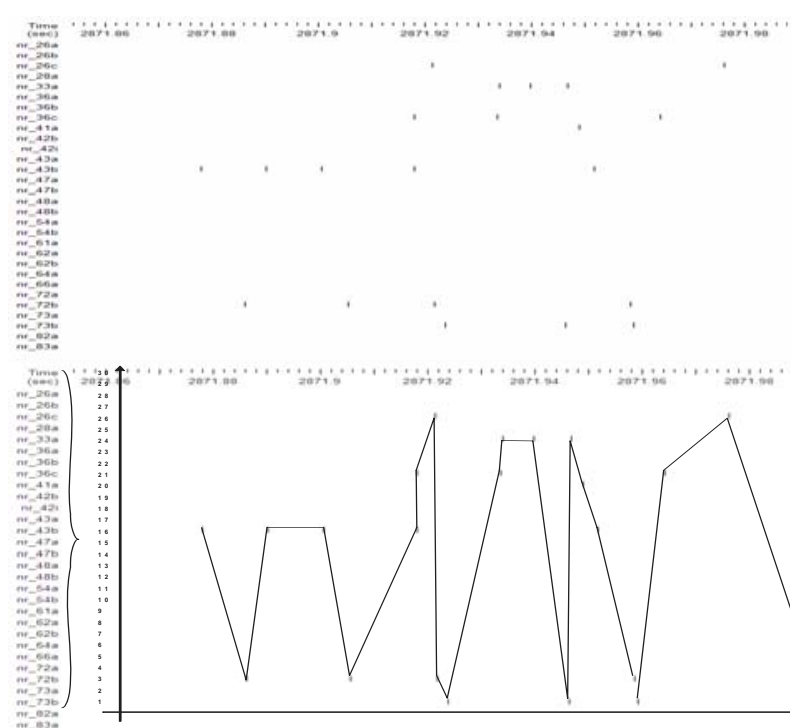

Fig.2: Graphic representation of the Space-Amplitude transform. In this way it can be thought as the interpolation of a wave(bottom) from the raster plot (top). This is obviously true for a linear traditional channel arrangement.

Starting from the obtained signal, for example, the repetitive pattern recognition task (as described in the INTRODUCTION) is reduced to a trivial $1 \mathrm{D}$ Autocorrelation computation on the data set or to a Cross correlation between a desired data sub-sequence, for example a Burst, and the data set.

Moreover, the chose of different arrangement algorithms, R, allows to evaluate different aspects of the network behavior. For example, after the calculation of the Auto or Cross correlation function of a Burst, as described above, the influence of the channel spatial arrangement can be investigated. In this perspective, the comparison can be done using a standard arrangement algorithm and a "Hilbert wave" like arrangement, that strongly respects the original topologic proximity, as shown in Fig.3:

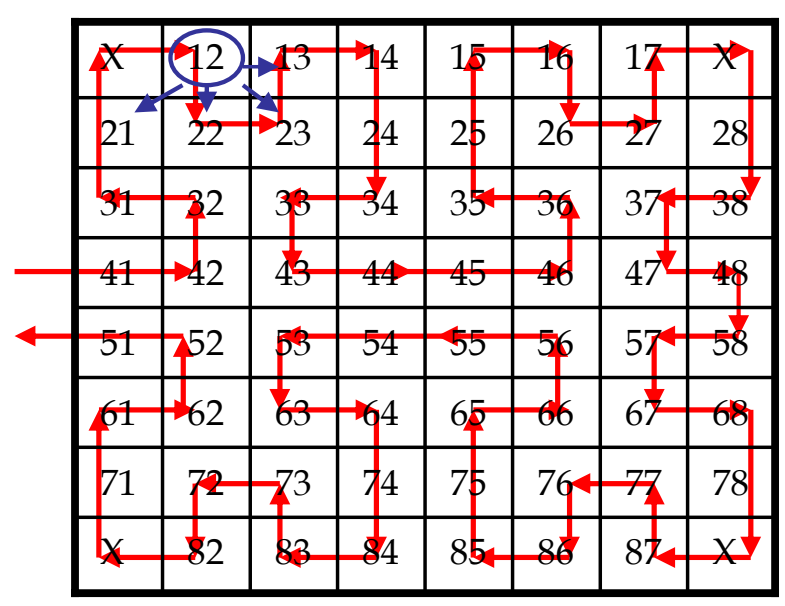

HILBERT-I.IKE ARR ANGEMENT

Fig.3: Two different kind of arrangement algorithms. The traditional one on the left and the Hilbert-like one on the right. 

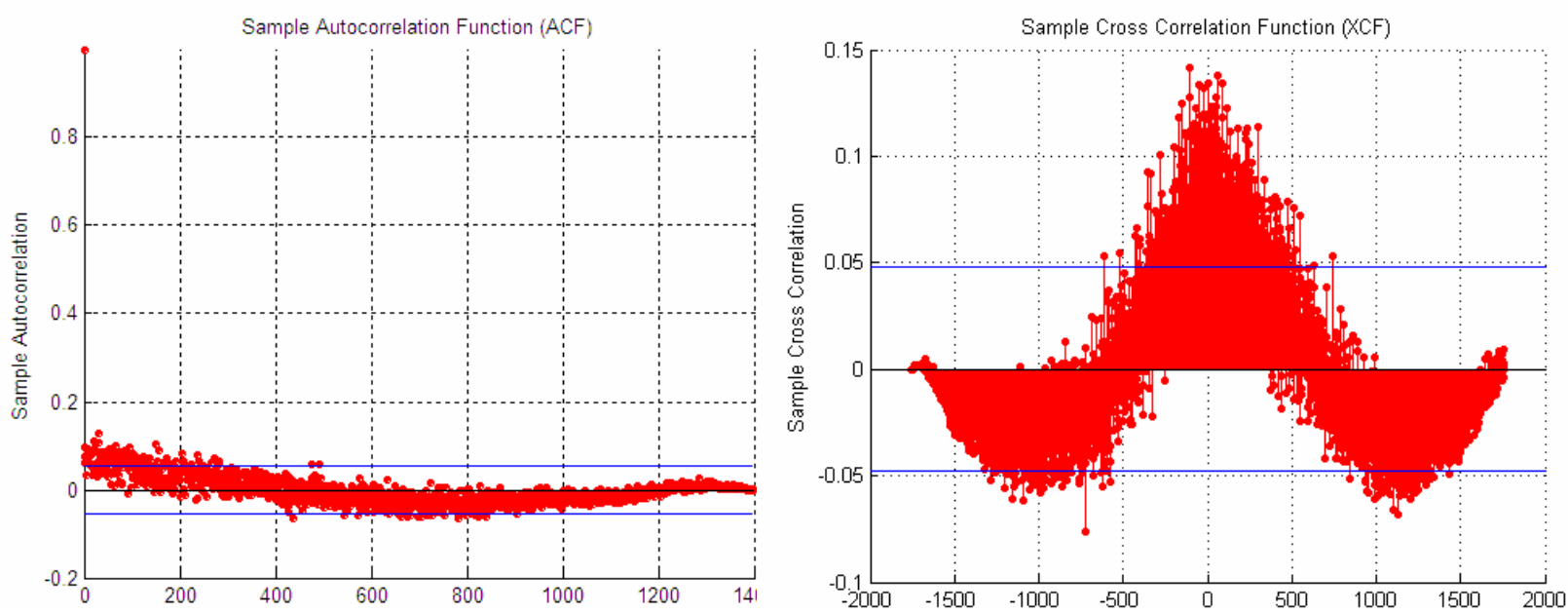

Fig.4: on the left: example of the Auto-correlation function (ACF) of a Burst Space-Amplitude representation. On the right: example of Crosscorrelation analysis of two distinct Bursts. The resolution is $0.1 \mathrm{~ms}$.

\section{RESULTS}

The method, as said, produces a 1D track, or a series of short 1D tracks. These tracks can be processed as an usual one-dim signal with consolidate signal processing methods. As an example, we show here same yet unpublished example of the proposed analysis. Fig.4 shows the standard linear processing analysis methods, i.e. Autocorrelation function (ACF) and Crosscorrelation function (CCF), of a single Burst (or a pair of Bursts in CCF) in Space-Amplitude representation (resolution $0.1 \mathrm{~ms}$ ).

Starting from CCF we can, for example, compare burst populations [16], i.e. series of bursts in a continuous track. It allows easily individuate groups of Bursts with similarities in their spike sequences (Fig.5).

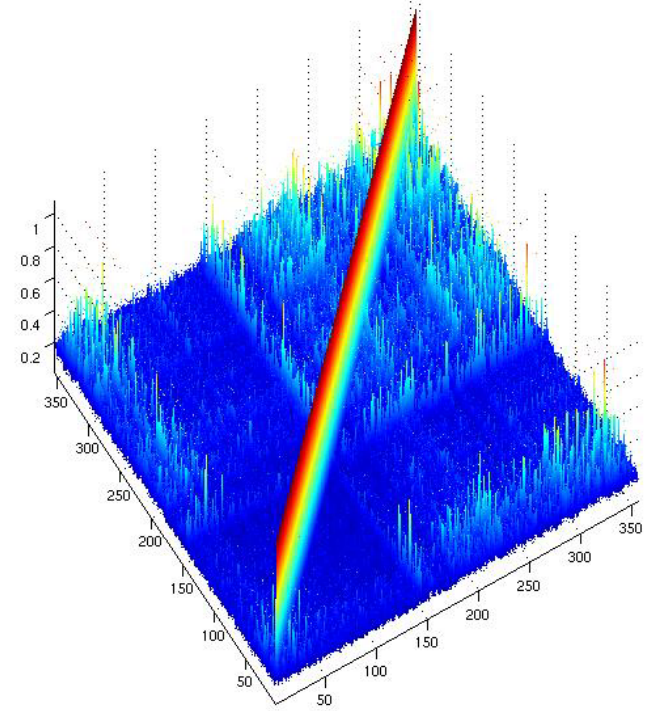

Fig.5: Plot of the correlation among 350 subsequent bursts of a MEA recording. In this plot is very easy to highlight groups of Bursts that have a similar spatio-temporal spike sequence.

We finally underline that the computational effort of these analysis is negligible, approximately in the order of 1 or 2 seconds of elaboration with a standard personal computer.

\section{DISCUSSION}

The method proposed allows to approach a very complex signal, as the raster plot point process, as a one-dimensional signal and to perform in a simplest way a simultaneous time-and-space analysis of a neuronal network. Because of the method involves a temporal sampling, an excessively low resolution can lead to computational problems. In fact too big temporal windowing could code some spikes as simultaneous. For this reason the best use of this algorithm is in high resolution analysis, e.g. with a resolution $<1 \mathrm{~ms}$. This high resolution approach is, for example, ideal for the intra and inter Burst analysis [17, 18].

Starting from a 1D signal, the Burst detection task becomes a trivial frequency analysis with an appropriate threshold value.

\section{CONCLUSIONS}

In conclusion we can summarize that the method presented here is a valuable tool for the high frequency neuronal network data analysis. It is characterized by a low computational effort. It allows to use consolidate analysis methods and multi-scale analysis and it is ideal for simple Burst detection algorithms. Furthermore it is completely invertible allowing a simple amplitude-space back-transformation.

Results obtained applying the proposed method on different kind of neuronal MEA datasets are encouraging. They'll be presented in following papers.

\section{ACKNOWLEDGEMENTS}

We thanks professor E. Wanke and his group of the Università degli Studi, Milano Bicocca, Dipartimento di Biotecnologie e Bioscienze, for the MEA data used, as examples, in this paper. 


\section{REFERENCES}

[1] U. Egert, B. Schlosshauer, S. Fennrich, W. Nisch, M. Fejtl, T. Knott, T. Muller and H. Hammerle, "A novel organotypic long-term culture of the rat hippocampus on substrate-integrated multielectrode arrays," Brain Research. Brain Research Protocols, vol. 2, pp. 229, 1998.

[2] S. M. Potter, "Distributed processing in cultured neuronal networks," Prog. Brain Res., vol. 130, pp. 49-62, 2001.

[3] M. S. Lewicki, "A review of methods for spike sorting: the detection and classification of neural action potentials." Network, vol. 9, pp. R53-78, 1998.

[4] E. N. Brown, R. E. Kass and P. P. Mitra, "Multiple neural spike train data analysis: State-of-the-art and future challenges," Nat. Neurosci., vol. 7, pp. 456-461, 2004.

[5] C. R. Legéndy and M. Salcman, "Burst and Recurrences of Bursts in the Spike Trains of Spontaneously Active Striate Cortex Neurons," J Neurophysiol, vol. 53, pp. 926-938, April. 1985.

[6] E. Persi, D. Horn, R. Segev, E. Ben-Jacob and V. Volman, "Neural modeling of synchronized bursting events," Neurocomputing, vol. 58-60, pp. 179-184, 2004.

[7] R. M. Davies, G. L. Gerstein and S. N. Baker, "Measurement of time-dependent changes in the irregularity of neural spiking," Journal of Neurophysiology, vol. 96, pp. 906, 2006.

[8] M. Abeles and G. L. Gerstein, "Detecting Spatiotemporal Firing Patterns Among Simultaneously Recorded Single Neurons," J. Neurophysiol., vol. 60, pp. 909-924, 1988.

[9] P. Parodi, Y. Jimbo, A. Kawana, D. MacRi and V. Torre, "Segmentation of the response of a neuronal network into clusters with similar activity," BioSystems, vol. 48, pp. 171-178, 1998.

[10] Y. Prut, E. Vaadia, H. Bergman, I. Haalman, H. Slovin and M. Abeles, "Spatiotemporal Structure of Cortical Activity: Properties and Behavioral Relevance," J Neurophysiol, vol. 79, pp. 2857-2874, June 1. 1998.

[11] I. V. Tetko and A. E. P. Villa, "A pattern grouping algorithm for analysis of spatiotemporal patterns in neuronal spike trains. 1. Detection of repeated patterns," J. Neurosci. Methods, vol. 105, pp. 1-14, 2001.

[12] I. V. Tetko and A. E. P. Villa, "A pattern grouping algorithm for analysis of spatiotemporal patterns in neuronal spike trains. 2. Application to simultaneous single unit recordings," J. Neurosci. Methods, vol. 105, pp. 15-24, 2001.

[13] E. M. Izhikevich, J. A. Gally and G. M. Edelman, "Spike-timing dynamics of neuronal groups," Cerebral Cortex, vol. 14, pp. 933-944, 2004.

[14] T. Hermle, C. Schwarz and M. Bogdan, "Employing ICA and SOM for spike sorting of multielectrode recordings from CNS," Journal of Physiology Paris, vol. 98, pp. 349-356, 2004.

[15] J. M. A. Tanskanen, J. E. Mikkonen and M. Penttonen, "Independent component analysis of neural populations from multielectrode field potential measurements," J. Neurosci. Methods, vol. 145, pp. 213-232, 2005.

[16] R. Segev, I. Baruchi, E. Hulata and E. Ben-Jacob, "Hidden neuronal correlations in cultured networks." Phys. Rev. Lett., vol. 92, pp. 118102\%N 11, 2004.

[17] W. Bialek, F. Rieke, De Ruyter Van Steveninck,R.R. and D. Warland, "Reading a neural code," Science, vol. 252, pp. 1854-1857, 1991.

[18] R. R. D. R. Van Steveninck, G. D. Lewen, S. P. Strong, R. Koberle and W. Bialek, "Reproducibility and variability in neural spike trains," Science, vol. 275, pp. 1805-1808, 1997. 